\title{
Rapid development of semantic 3D city models for urban energy analysis based on free and open data sources and software
}

\author{
JOCHEN WENDEL, \\ ALEXANDER SIMONS, \\ ALEXANDRU NICHERSU, \\ SYED MONJUR MURSHED, \\ European Institute for Energy Research (EIFER), Karlsruhe Institute of Technology, Karlsruhe, Germany
}

\begin{abstract}
Geospatial data, specifically semantic 3D building data, plays a crucial role in urban energy analysis as spatial calculations using 3D geometries usually form the basis for energy simulation and modelling needed for numerous smart cities applications. Additional information describing the building stock, such as building materials and energetic properties but also data not directly linked to urban morphology such as weather data, environmental data, vegetation or socio-demographic data sets are required for these applications. A major drawback in the widespread applicability of urban energy analysis is the lack of available data sets as well as the costly and lengthy labor-intensive process of generation of those data sets (e.g. 3D city models or LIDAR data). While recent years have seen an opening up of urban data sets through free and open data portals, web services, and APIs that are used for urban energy analysis, data standardization and varying data quality still raises big challenges. This research explores different methodologies for the generation and usage of semantic 3D city models based on free and open data sources and software. In this paper, we describe four different methodologies for the generation of semantic 3D city models from available open data (geospatial data portals, LIDAR data, Open Street Map data, and remote sensing data) and the tools required to achieve the task. To evaluate the suitability of these open-data sets for smart cities applications, multiple energy models, such as an energy performance model and a vertical solar radiation tool, previously developed in EIFER, have been applied to evaluate the applicability of these generated city models.
\end{abstract}

\section{KEYWORDS}

3D city models, CityGML, Urban energy analysis, Free and open data and software, Smart and sustainable cities

\section{INTRODUCTION}

Virtual 3D city models have a long history in the application of different types of complex urban phenomena. Most of the 3D city

Permission to make digital or hard copies of all or part of this work for personal or classroom use is granted without fee provided that copies are not made or distributed for profit or commercial advantage and that copies bear this notice and the full citation on the first page. Copyrights for components of this work owned by others than the author(s) must be honored. Abstracting with credit is permitted. To copy otherwise, or republish, to post on servers or to redistribute to lists, requires prior specific permission and/or a fee. Request permissions from Permissions@acm.org.

UrbanGIS'17, November 7-10, 2017, Redondo Beach, CA, USA

(C) 2017 Copyright is held by the owner/author(s). Publication rights licensed to ACM.

ACM 978-1-4503-5495-0/17/11 ..\$15.00

https://doi.org/10.1145/3152178.3152193 models, purely based on graphical and geometrical models, were developed for visualization purposes to ensure a photorealistic resembling of visual urban objects. In addition, recent developments focused on the inclusion of semantical aspects that are suitable for modelling and analysis purposes. For example, with the development and introduction of standardardized data models such as CityGML, one can not only represents the graphical appearance of city models, but also include the representation of semantics, thematic properties, taxonomies and aggregations of different features (e.g. walls, roofs, etc.). Such virtual 3D city models form a basis for integrating numerous other information (e.g., building installation) and data (e.g. building energy characteristics) within a single framework at the different Level of Details (LoD) [1]. This standard can be used as key inputs in the context of smart and sustainable cities services [2-3]. Examples in an urban context can be found in many different domains such as noise simulation, telecommunication planning, real time monitoring, sensor data placement and integration, indoor navigation and building energy modelling, where virtual 3D city models play a major role. Biljecki et al. [4] provide a comprehensive state of the art review of applications for 3D city models were over 100 models and 29 use cases are defined.

Buildings contribute to approximately $40 \%$ of the total energy consumption of a city. Therefore, the detailed 3D information on the building stock will ensure more accurate urban energy analysis [5]. This can help local governments to meet national and international climate goals. For example, through the geometric calculation based on the 3D building models and other building physical characteristics, dynamic heating and cooling demands for buildings can be estimated accurately [6-7]. Furthermore, some analyses such as incoming solar energy radiation or technoeconomic PV potential can be accurately calculated even at finer scale, i.e. roof or wall surface level. They can be further aggregated to building or neighborhood level. In order to carry out such analyses, semantic 3D models of higher grade of representation are required [8].

The number of cities represented in 3D city models is increasing rapidly, while the benefits are apparent and the investment costs and time required to build these models are decreasing as new automatic data collection technologies such as LIDAR are developed. However, the generation of city wide buildings models 
Wendel, J. et al.

is still costly and time consuming and making the widespread applicability of energy analysis on a city wide scale only in a few cases feasible. In recent years there has been a push from local governments through larger programs such as INSPIRE in Europe or the open data initiatives in many North American cities to provide open data sets that also include 3D building data. However, 3D models vary in data quality and there is a slow adoption of standards, which hinders the widespread use of such models.

Therefore, the main objective of this research is to develop methodologies to generate 3D city models, on the basis of free and open data and software that is suitable for energy analysis and modelling and not limited to any geographic region. Moreover, the rapid generation should be of low cost and could be of lower level of details 3D city models that would be sufficient for an energy analysis.

\section{CITYGML A STANDARD FOR SEMANTIC 3D CITY MODELS}

In this research, CityGML is used as the data format for the representation of 3D building data due to its widespread usage and being an open data standard. While other data formats such as $\mathrm{X}_{3} \mathrm{D}^{2}, \mathrm{COLLADA}^{3}$ and KML also allow the inclusion of semantic information, there is no true standardization on how representation of complex geographic features and their interrelationships are handled [1].

CityGML is an international Open Geospatial Consortium (OGC) standard for the representation and exchange of semantic 3D city and landscape models adopted. The data model behind CityGML is based on the ISO 19100 standards family and is implemented as an application schema for OGC's Geography Markup Language (GML) [9-10]. The CityGML standard allows the possibility of extending the standard by Application Domain Extensions (ADE). For energy modelling purposes, ADEs such as the Energy $\mathrm{ADE}^{4}$ and the Utility Network $\mathrm{ADE}^{5}$ are of great value, as they allow standardized data storage and exchange of energy relevant parameters directly in the CityGML data model. Furthermore, object modelling specifications can be shown at different Levels of Detail (LoD) ranging from simple building block models (LoD1) to full detailed building models that include building interiors (LoD4). Only the generation of LoD1 and LoD2 models from free and open data sources are considered as these LoDs are also the most common levels for representing a city wide buildings models.

\section{RESULTS AND DISCUSSION}

The successful deployment of energy models requires a flexible data infrastructure that allows adaptability, reusability and open-

\footnotetext{
${ }^{2}$ http://www.web3d.org/x3d/what-x3d

${ }^{3}$ https://www.khronos.org/collada/

${ }^{4}$ http://www.citygmlwiki.org/index.php/CityGML_Energy_ADE

5 http://www.citygmlwiki.org/index.php?title=CityGML UtilityNetworkADE
}

standards to manipulate and fine tune code as well as to act as a central hub that allows the connection of data from different domains. For efficient central data storage of information from all energy models, sensors and spatial analysis tools, the 3DCityDB, a simplified CityGML database schema is used [11]. In addition, the usage of PostGIS within this infrastructure enables the usage and handling of spatial functions that are required for visualizing model outputs at different visualization modes (Fig. 1).

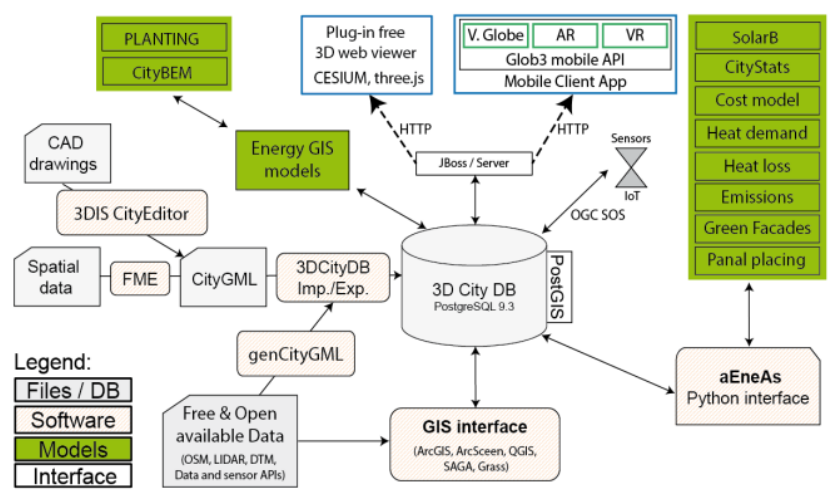

Fig. 1. Open-source data infrastructure for urban energy analysis.

The open-source software infrastructure consists of data preparation, quality check, storage, analyses and visualization interfaces (PostgreSQL,3DCityDB, PostGIS). Different urban energy analyses tools are integrated into a python interface, called aENeAs. For example, SolarB performs city wide computations of solar radiation received by buildings' wall and roof surfaces [12], CityStats performs a socio-demographic clustering model for energy usage [13], CityBEM calculates monthly cooling and heating energy need of the buildings [6], heat demand and heat loss model calculate annual heat demand [11]. Furthermore, multiple visualization interfaces including 3D web mapping (three.js, Cesium), Augmented reality (AR), Virtual Reality (VR) and mobile 3D mapping have been realized (Glob3m API) [1415]. This paper will focus on the generation of additional semantic 3D city models of other cities from free and open data that can be used by the above mentioned software infrastructure.

\section{COMMON PROBLEMS IN SEMANTIC 3D CITY MODELS}

Different requirements for 3D city models for visualization and modelling exist. The models for visualization purposes do not need to have geometrically correct surface models, but the analysis and simulation of energy related issues require geometrical and topological correct surfaces. Many 3D city models, especially the once automatically generated from LIDAR or by photogrammetric methods lack correct topology and geometry. Beljeki et al. [16] reviewed the common problems associated with the CityGML data across different cities. We found some most common errors during the review and 
preparation of CityGML datasets. The errors originate due to wrong definition of normal vectors, wrong definition of building installation parts (such as building extensions should be defined as separate geometry object) and non-planarity of building surface. Due to these problems, the energy models are not able to run on the concerning buildings.

The determination of normal vectors of surfaces, surface area, surface orientation or the slope of the geometry object are required. Fig. 2 depicts a point grid that was distributed over different surfaces of a building. It resembles solar radiation values calculated by the SolarB tool. The point grid is generated in a plane and becomes rotated into the position of the surface by using its computed normal vector. In this case the calculation of the normal vector failed for the surfaces with the ID 2, 4 and 8, because of redundant points, that where in-between the corner points of the polygons (extreme points that define the plane). The surfaces 2 and 4 were corrected in the PostgreSQL database by the PostGIS function ST_SimplifyPreserveTopology, which is an implementation of the Douglas-Peucker-Algorithm. However, this approach does only work for geometries that are oriented horizontally in their execution. In our analyses, multiple 3D city models were encountered showing this problem and making them unusable for energy modelling.
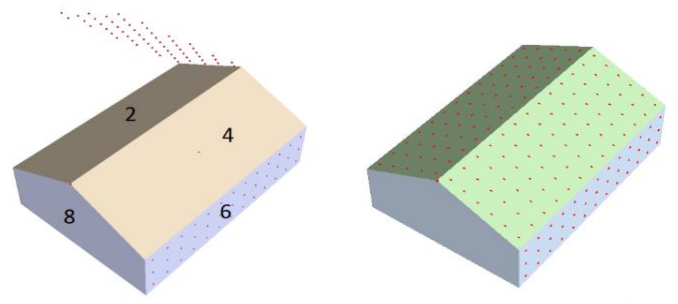

Fig. 2. Surface 2 and 4 show non-planarity of surfaces, the energy model outputs are aligned along the wrongly defined normal vector (left), building surfaces are aligned along the normal vector of the surface after correction (right)

Some other problems are more difficult to correct and in most cases require significant amount of time to generate a rectified city model (e.g. nesting of polygons within one surface). Generation of simplified 3D city models (LoD1 and 2) from open sources are preferred and serves the requirements for energy analyses.

\section{OPEN DATA FOR 3D CITY MODELS}

The availability of ready to use (i.e. semantically and topologically correct) open 3D city models varies heavily among the geographic regions. In recent years, many cities and states, especially in Europe and North America have made their 3D data sets accessible to the public. Most of them are only provided in LoD1 but some LoD2 data sets are starting to appear [4]. For example, several federal states in Germany such as North Rhine-
Westphalia ${ }^{6}$ and Thuringia ${ }^{7}$ published their whole 3D building model data sets covering the whole state in LoD1 and partially in LoD2 in the CityGML format in early 2017. Also in North America, New York $^{8}$ and Montréal ${ }^{9}$ provided good quality data sets in LoD1 and LoD2. Besides the CityGML format, multiple cities also provide their LoD1 and LoD2 3D data in other formats such as KML, multi-patch SHP, Blender or GeoJSON. A complete and updated list of cities providing $3 \mathrm{D}$ data is available on the CityGML ${ }^{10}$ website.

Besides ready to use 3D city models, many free and open LIDAR data sets are available. Some countries such as Canada, Denmark, Finland, Latvia, Netherlands, Poland, Slovenia, Spain, Sweden, Switzerland, USA, England and New Zealand provide national LIDAR data set of varying coverage and quality. These data sets can be used as input to generate city models or to derive building height values when only building footprint data is available. Multiple methods (Section 5.1) and open-source software such as 3 difer ${ }^{11}$, to generate semantic 3D city models exist.

However, many cities still do not have any official 3D city models. OpenStreetMap (OSM) is an alternative for the creation of $3 \mathrm{D}$ city models. OSM describes different tags to illustrate basic attributes of buildings, and according to Goetz and Zipf [17] nearly $90 \%$ of buildings in Germany should be covered at least by footprint information. For example, building and building parts can be tagged with a height, roof shape, roof material, etc. By using this tag, even LoD2 models can be generated if OSM tags regarding roof shapes are present. Otherwise, the building height or the number of building levels could be used to generate LoD1 models. Multiple tools, such as OSMbuildings ${ }^{12}$ exist to check whether 3D building information is available in the area of interest. However, OSMbuildings and other 3D visualization tools for OSM tend to assign default building heights of $3.5 \mathrm{~m}$ to buildings that have no height values tagged.

\section{METHODOLOGY}

The generation of semantic 3D city models (CityGML) from open data can be performed mainly through four different methods: (a) using available data of open data portals (e.g. New York City), (b) using open LIDAR data and tools for the creation of semantic 3D city models (e.g., Zurich), (c) using OSM data to generate a city model (e.g. Hong Kong), and (d) using building footprint and remote sensing data (e.g. Karlsruhe, Germany) are presented. Fig. 3 explains different steps and tools associated with these methods to generate CityGML data sets.

\footnotetext{
${ }^{6}$ https://www.opengeodata.nrw.de/produkte/geobasis/3d-gm/ ${ }^{7}$ http://www.geoportal-th.de/dede/downloadbereiche/downloadoffenegeodatenth\%C3\%BCringen/download3dgeb\% C3\%A4ude.aspx

${ }^{8} \mathrm{http}: / / \mathrm{www} 1$.nyc.gov/site/doitt/initiatives/3d-building.page

${ }^{9} \mathrm{http}$ ://donnees.ville.montreal.qc.ca/dataset/maquette-numerique-batiments-citygmllod2-avec-textures

${ }^{10}$ https://www.citygml.org/3dcities/

11 https://github.com/tudelft3d/3dfier

12 https://osmbuildings.org/
} 


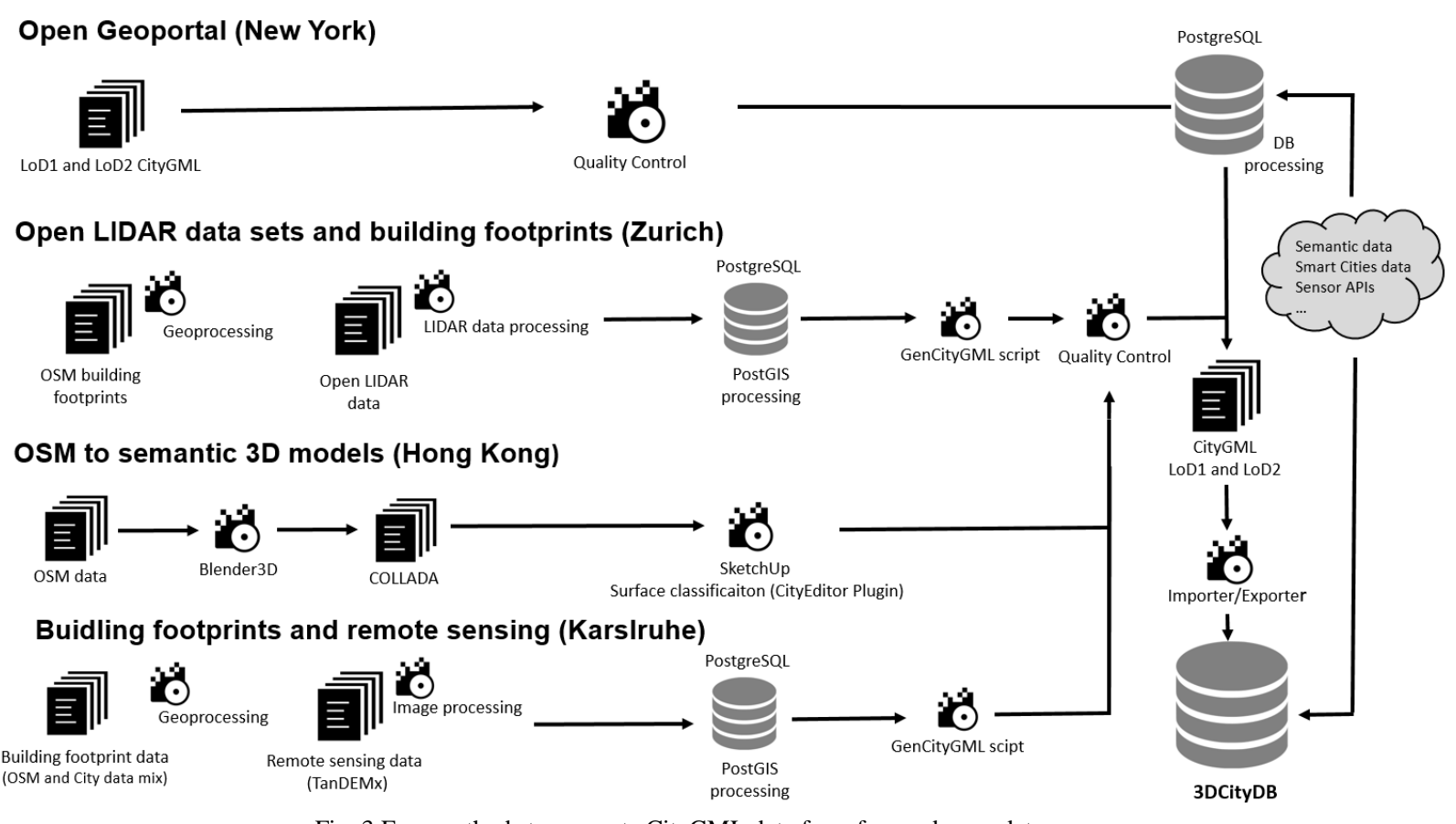

Fig. 3 Four methods to generate CityGML data from free and open data sources

Open geoportal: The New York City open data portal is the most prominent example, offering over 1300 free and open data sets that also include a building data set with 1.5 million buildings in the CityGML LoD1 and LoD2 format. After quality checks (geometrical, topological and attributes), this data can be directly imported into our software architecture (Fig. 2) to perform energy analysis. In addition to the building data, other data sets, such as semantic building data, transportation data and open weather data can also be integrated directly into the 3DCtiyDB as generic attributes or via prior database processing directly into the CityGML format and its ADEs.

Open LIDAR data sets and building footprints: LIDAR data is combined with OSM footprint data to extract the height information from the LIDAR data set. As a test case, the city of Zurich in Switzerland has been chosen due to the availably of an open national LIDAR database. Depending on the resolution of the LIDAR data set, LoD1 and LoD2 models can be generated. In this approach, we have adopted a methodology proposed by Fernández ${ }^{13}$ using LAStools and PostGIS functions for extracting height information of buildings from LIDAR data. This is done by generating building buffers to calculate a mean average height from the LIDAR points. For LoD1 models, the average height value from the buffer analysis is taken to define the building height, for LoD2 models a ridgeline is identified in the LIDAR point cloud and averages values are taken along this ridge are taken to define the roof height and shape. Moreover, a custom

$13 \mathrm{https} / / / \mathrm{www}$. altergeosistemas.com/blog/2015/04/30/extrayendo-la-altura-de-losedificios-a-partir-de-datos-LIDAR/ script (genCityGML, available in GitHub ${ }^{14}$ ) is developed to generate LoD1 and LoD2 CityGML standard from the 2D geometries by adding height information of ground and roof surfaces. The script is based on the Random3Dcity ${ }^{15}$ tool developed by the TU Delft.

OSM to semantic 3D models: The city of Hong Kong has been chosen to test a methodology for the generation of CityGML LoD1 and LoD2 from OSM data. In this approach, the open source Blender plugin blender-osm ${ }^{16}$ has been used. It allows importing OSM building data and terrain into blender. In addition, it supports the generation of multiple roof types (e.g. flat, gabled, hipped, dome) that are relevant for energy analysis and modelling. In order to generate CityGML from Blender, a surface type classification of the building surfaces has to be conducted. Therefore, the data has to be exported to SketchUp in the COLLADA format. COLLADA proofed to be the most stable solution and is in favour of 3DS that generated inconsistent scaling and unit conversion errors. The 3D data can then be directly exported as CityGML from SketchUp using the CityEditor ${ }^{17}$ plugin and, after quality checks, imported into the 3DCityDB.

Building footprints and remote sensing data: In this approach, OSM building footprint data from the city of Karlsruhe, in conjunction with TanDEM-X ${ }^{18}$ (TerraSAR add-on for Digital

\footnotetext{
${ }_{15}^{14}$ https://github.com/Xandersimons/genCityGML

${ }_{15}$ https://github.com/tudelft3d/Random3Dcity

${ }_{16} \mathrm{http} / / /$ wiki.openstreetmap.org/wiki/Blender

${ }^{17} \mathrm{https://www.3dis.de/loesungen/3d-stadtmodelle/cityeditor/}$

18 https://tandemX-science.dlr.de/
} 


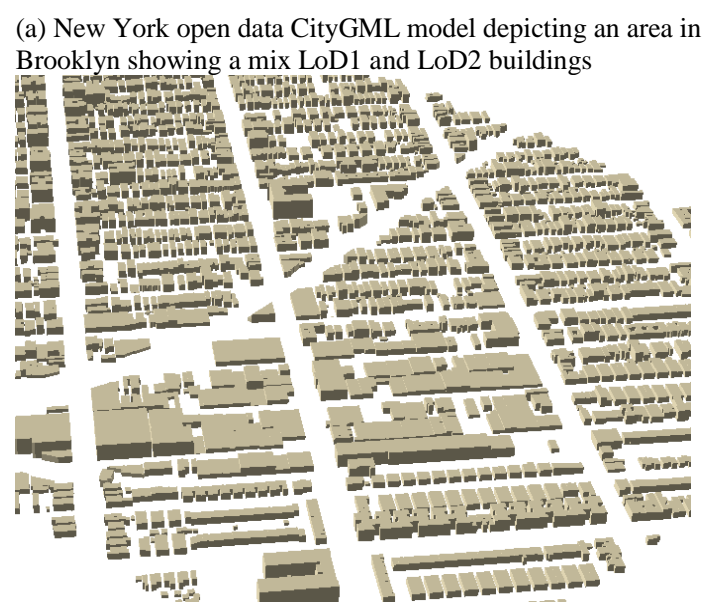

(c) Lod 2 model of downtown Hong Kong (Bank of China) obtained from OSM via Blender

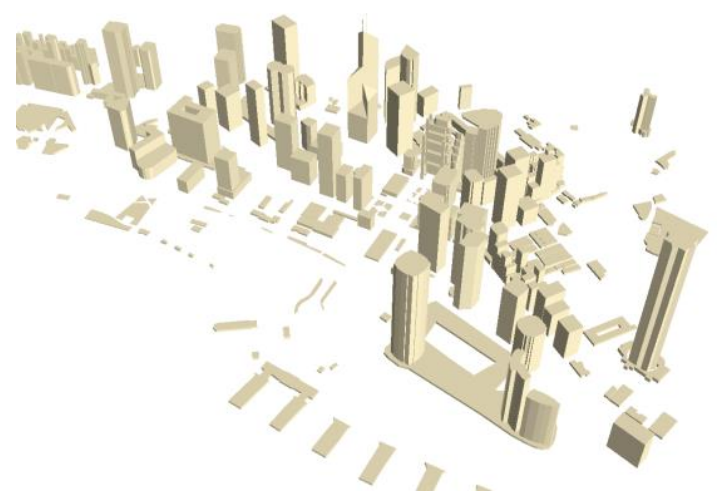

b) A subset of the Zurich CityGML Lod1 model derived from LIDAR data

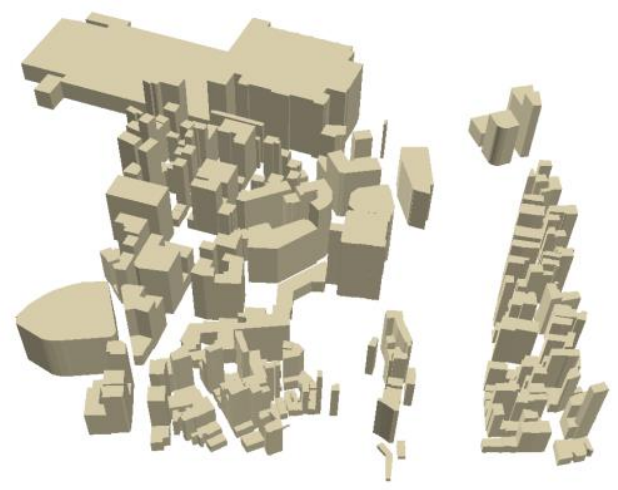

(d) City wide Lod1 and Lod2 model of Karlsruhe derived from building footprint and remote sensing data

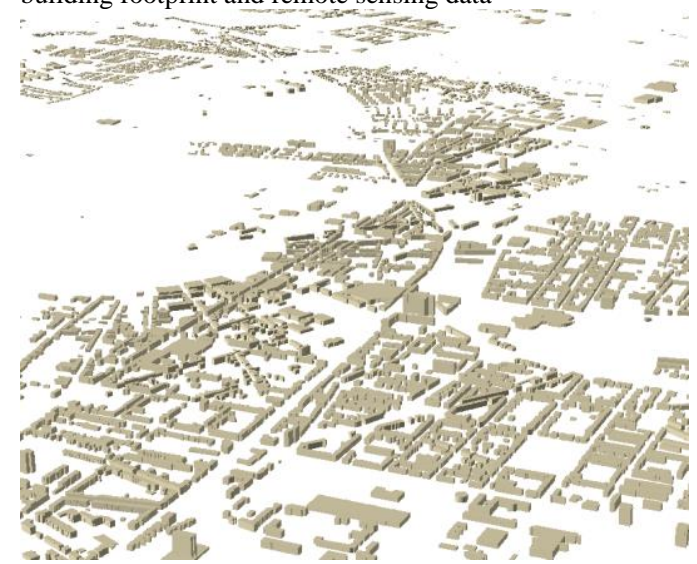

by the four different methodolc
Elevation Measurements) remotely sensed data, was used to generate 86.000 buildings. The buildings were modelled in LoD1 as well as some selected buildings in LoD2. The height of each building was estimated by the number of floors of each building that was estimated from the TanDEM-X data. For each floor a standard height, value in meters, depending on the building type, was used to estimate the total height of a building. This method also exploits the genCityGML script to generate the CityGML standard.

\section{RESULTS}

All methodologies are able to produce topological and geometrical correct CityGML models. In the first method, after quality control and geometric corrections the CityGML the data set from the New York open data portal was ready for import to the 3DctiyDB. It was the most efficient way (Fig. 4 (a)). Quality control is most important in the generation processes of the $3 \mathrm{D}$ city model for Zurich, as several instances of non-planar surfaces were encountered due to the automatic delineation of the height attributes from the LIDAR data (Fig. 4 (b)). The third methodology is using Blender and OSM to create semantic 3D city models. It was the most resource and time intensive method. While the direct import of OSM data into Blender is tempting, especially for visualization purposes, it is not optimally suited for the generation of semantic 3D model. Automatic identification of surface types is not possible in all cases and surface types have to be assigned manually. Furthermore, topological errors and missing or disconnect surfaces are observed. (Fig. 4 (c)). The generation of the 3D model for Karlsruhe using TanDEM-X data is able to produce a city wide model in LoD 1 and LoD 2. However, manual intervention is still necessary, as it was not possible to estimate the building height in all cases. One reason is that the TanDEM-X data was still in an experimental stage when the 3D city model was derived [18] (Fig. 4 (d)).

For evaluation of the applicability of energy models, several energy models and applications are successfully applied. Fig. 5 shows two different examples of energy relevant applications. Fig. 5 (left) shows the successful application of the SolarB tool to illustrate solar radiance values applied on a LoD2 model of Staten Island. Besides the building data, additional open-data such as 

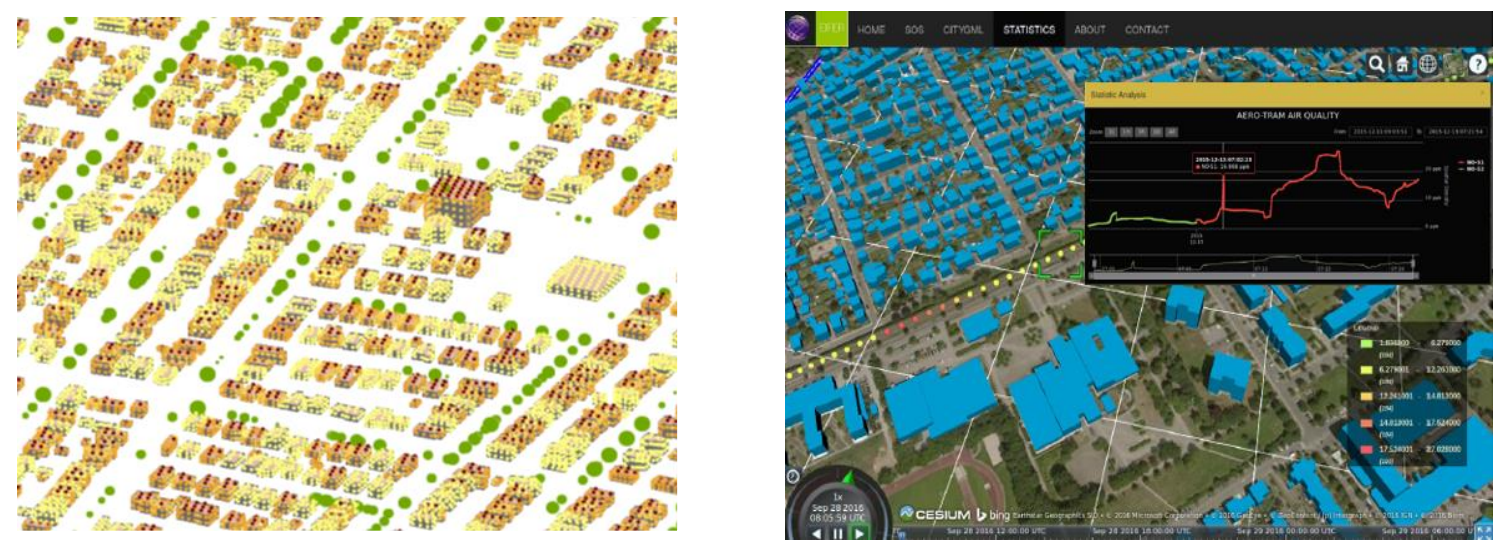

Fig. 5 SolarB model outputs and open vegetation data applied to a subset of Staten Island in New York (left), mobile sensor data from AeroTram integrated showing fine particle matter pollution in the LoD1 3D city model of Karlsruhe in Germany (right)

weather data from OpenWeatherMap 19 and Weather Underground ${ }^{20}$ as well as information on trees from the New York open data portal are integrated into the model. Fig.5 (right) shows the LoD1 model of Karlsruhe integrated with mobile sensor data from the AeroTram ${ }^{21}$ project measuring environmental data along the tracks of a local tramline. In this proof of concept, sensor data is integrated via OGC SOS (Sensor Observation Service) with the CityGML data [19].

\section{DISCUSSION AND CONCLUSION}

In this paper, we have described four different methodologies for the rapid generation of semantic 3D city models from available open data sources. Although the current situation needs some improvement regarding data availability, there is an obvious trend showing cities are allowing their data to become publicly available. This allows implementing and testing the four methods presented in this paper.

The open data portal of New York represents the ideal example for free and openly available 3D building data. It generates great value to researchers, individuals, public and private companies. In the context of smart cities, many large cities have taken this path, with examples varying from Berlin, Lyon, and Rotterdam to New York. In the case of the second method, we explored the suitability of LIDAR data sets for the generation of 3D models. Many cities and states perform aerial surveying using drone or airplanes of their territories on a regular basis, making this data source very promising when it is combined with building footprints and other information. The process, although requires additional processing, geometry and topology checks are mandatory for semantical correct building models. The third methodology converts OSM data to COLLADA. This method requires that the OSM data set contain $3 \mathrm{D}$ information that is not

\footnotetext{
${ }^{19}$ https://openweathermap.org/

${ }^{20}$ https://www.wunderground.com/

${ }_{21}$ http://www.aero-tram.kit.edu/
}

always the case with open data, as is the case of OSM. This method proved to be excellent for creating 3D models for visualization purposes (e.g. Blender) but it has the disadvantage of requiring manual work efforts and rigorous geometry and topology checks for conversion to a semantical city model. For the fourth use case, we used building footprints from OSM and remote sensing data from the TanDEM-X satellite. Due to its high temporal resolution, this method is very suitable for areas affected by fast and large scale change, such as conflict zones or zones affected by natural disasters where a large number of structure have been razed to the ground but the footprint information still exists in OSM data sets. This method allows the reconstruction effort to be very well estimated and supported.

When considering the significance of 3D models on urban energy analysis we see that the availability of semantical city models is a pre-requisite for analyses such as solar irradiation or building energy needs. Different geometrical and statistical analyses such as sky view factor, shadowing, surface to volume calculations can be performed more accurately with the 3D city models. They in turn, with the combination of robust algorithms and other data sets (e.g. building attributes, weather data, etc.), perform rigorous energy analyses. At the same time, these spatial models have become an integral point for the merging of multiple data sources (socio-economic indicators) allowing for spatial clustering, see [13]. While the data quality varies, we have experienced the data to be excellent for fast prototyping or the generation of proof of concepts. Furthermore, while there is still unknown uncertainty in most of the open 3D models, we found these models extremely valuable for the generation of a top-down approach, where in a first step analysis in conducted on a coarser city wide scale to identify potential issues and then in a second step finer scale data is used to analysis these issues.

While standardized web services for IoT and smart cities such as the OGC SWE ${ }^{22}$ (Sensor Web Enablement) tool kit for

\footnotetext{
${ }^{22}$ http://www.opengeospatial.org/ogc/markets-technologies/swe
} 
Wendel, J. et al.

standardized sensor data exchange or FIWARE ${ }^{23}$, a holistic approach towards smart cities, are already well established, it is the $3 \mathrm{D}$ city models that binds the sensors and physical devices together. Devices, vehicles and people are connected not just trough IT systems but also by the environment (be it built or natural) they share and one of the best ways to quantify this influence for urban energy analysis is through 3D city models.

\section{ACKNOWLEDGMENTS}

Part of this research that involved the generation of the city model of Karlsruhe was supported by the German Federal Ministry for Economic Affairs and Energy grant \# [50EE1101]. We would like to thank Thomas Hauenstein from the Liegenschaftsamt of the city of Karlsruhe for providing us with extensive data samples for the evaluation of our methods and models. We would also like to thank our colleagues at the KIT-IPF for their support.

\section{REFERENCES}

[1] Kolbe, T. H., Gröger, G., Plümer, L. 2005: CityGML Interoperable Access to 3D City Models. In Proceedings of the first International Symposium on Geo-Information for Disaster Management, Springer.

[2] Becker, T., Nagel, C., \& Kolbe, T. H. 2011. Integrated 3D modeling of multi-utility networks and their interdependencies for critical infrastructure analysis. In Advances in 3D Geo-Information Sciences. Springer Berlin, Heidelberg, 1-20.

[3] Döllner, J., Baumann, K., \& Buchholz, H. 2006. Virtual 3D city models as foundation of complex urban information spaces. In Proceedings Sustainable Solutions for the Information Society 11th International Conference on Urban Planning and Spatial Development for the Information Society, 107-112.

[4] Biljecki, F., Stoter, J., Ledoux, H., Zlatanova, S., and Çöltekin, A. 2015. Applications of 3D city models: State of the art review. ISPRS International Journal of Geo-Information, 4 (4), 28422889.

[5] Bahu, J.-M., Koch, A., Kremers E. and Murshed, S. M. 2014 Towards a 3D spatial urban energy modelling approach. International Journal of 3-D Information Modeling (IJ3DIM), 3, 1-16.

[6] Murshed, S.M., Picard, S., Koch, A. 2017. CityBEM: An Open Source Implementation and Validation of Monthly heating and cooling energy needs for 3D Buildings in Cities. The ISPRS Annals of the Photogrammetry, Remote Sensing and Spatial Information Sciences (ISPRS Annals). In press.

[7] Agugiaro, G. 2016. Energy planning tools and CityGML-based 3D virtual city models: experiences from Trento (Italy). Applied Geomatics, 8, 41-56.

[8] Nouvel R., Zirak M., Dastageeri, H., Coors, V., Eicker, U. 2014. Urban Energy Analysis based on 3D City Model for National Scale Applications. In IBPSA Germany Conference. 83-90.

[9] Gröger, G. and Plümer. L. 2012. CityGML - Interoperable semantic 3D City Models. ISPRS Journal of Photogrammetry and Remote Sensing, 2012(71), 12-33.

[10] Kolbe, T. H. 2009). Representing and exchanging 3D city models with CityGML. 3D geo-information sciences, 15-31.

[11] Simons, A. and Nichersu, A. 2014. Development of a CityGML infrastructure for the imple-mentation of an energy demand method with different data sources. GIScience 2014.

[12] Wieland, M. Nichersu, A. Murshed, SM, and Wendel, J. 2015. Computing Solar Radiation on CityGML Building Data. In Proceedings of the 18th AGILE International conference on Geographic Information Science 2015. Lisbon, Portugal. 9th June 2015.

[13] Saed, S. and Wendel, J. 2015. Estimating heating energy consumption and $\mathrm{CO} 2$ production - A novel modeling approach. In Proceedings of the 14th International Conference on Computers in Urban Planning and Urban Management. Cambridge, MA.

[14] Santana, J. M., Wendel, J., Trujillo, A., Suárez, J. P., Simons, A., \& Koch, A. 2017. Multimodal Location Based ServicesSemantic 3D City Data as Virtual and Augmented Reality. In Progress in Location-Based Services. Springer, 329-353.

[15] Wendel, J., Murshed, S.M., Sriramulu, A. and Nichersu, A. 2016. Development of a Web-Browser Based Interface for 3D Data-A Case Study of a Plug-in Free Approach for Visualizing Energy Modelling Results. Lecture Notes in Geoinformation and Cartography, Springer, 185-205.

[16] Biljecki, F., Ledoux, H., Du, X., Stoter, J., Soon, K. H., \& Khoo, V. H. S. 2016. The most common geometric and semantic errors in CityGML datasets. ISPRS Annals of Photogrammetry, Remote Sensing \& Spatial Information Sciences, 4(2), 13-22.

[17] Goetz, M., and Zipf, A. 2012. OpenStreetMap in 3D-detailed insights on the current situation in Germany. In Proceedings of 15th AGILE International Conference on Geographic Information Science, 24-27.

[18] Thiele A., Wurth MM, Hinz S., and Even M. 2013. Extraction of Building Shape from TanDEM-X Data. International Archives of the Photogrammetry. Remote Sensing and Spatial Information Science. (Volume XL-1/W1): 345-350.

[19] Zhu, W., Simons, A., Wursthorn, S., \& Nichersu, A. 2017. Integration of CityGML and Air Quality Spatio-Temporal Data Series via OGC SOS. In Conference proceedings Geospatial Sensor Web Conference 2016. Münster, Germany.

\footnotetext{
${ }^{23}$ https://www.fiware.org/
} 